\title{
Stretching in the prevention of hamstring strains: Attitudes, beliefs and current practices among football coaches in Mauritius
}

\author{
Kelly Chan $^{1}$, Fawzi M. Mahomoodally ${ }^{1^{*}}$, Rajiv Veeren ${ }^{2}$ \\ ${ }^{1}$ Department of Health Sciences, Faculty of Science, University of Mauritius, Reduit, Mauritius; \\ *Corresponding Author: f.mahomoodally@uom.ac.mu \\ ${ }^{2}$ Ministry of Health and Quality of Life, Mauritius
}

Received 2 January 2012; revised 20 February 2012; accepted 5 March 2012

\section{ABSTRACT}

Context and Objective: Hamstring strain is a common injury in football and it causes a significant amount of time lost from competition and training. Since poor flexibility is thought to predispose to muscle strain, stretching is routinely recommended during warm-up routines by coaches to prevent injuries. However, available evidence suggests that pre-exercise stretching (PES), especially static stretching, has no benefit on injury rates and may even reduce performance in explosive type activities. We designed this study to assess the attitudes, beliefs and practices of football coaches regarding stretching in the prevention of hamstring strains. Design: A cross-sectional survey. Setting: Mauritius Football Association (MFA). Participants: 26 football coaches registered with the MFA. Intervention: Questionnaires were distributed to football coaches of the MFA via sports officers. Questionnaires were then collected two weeks after distribution. Main Outcome Measures: Attitudes, beliefs and practices of football coaches regarding stretching in the prevention of hamstring strains. Results: MFA coaches held generally positive attitudes and beliefs towards stretching. $88 \%$ of coaches felt that PES is beneficial and $93 \%$ believed that PES prevents hamstring strains. The majority of coaches recommended stretching after warming up (81\%) and after the training session (93\%). $76 \%$ of coaches also advised stretching outside the training sessions. $96 \%$ of coaches used static stretching to stretch the hamstrings. The hamstrings were stretched on average for 4 times at each training session and the mean duration of a static stretch was 12 seconds. Conclusions: Nearly all coaches believed that PES prevents hamstring strains although evidence is limited. Some of the coaches' beliefs and practices were not in line with current recommendations. Coaches reported that their stretching practices would be most likely influenced by scientific research. Thus there is an urgent need to devise awareness and training programmes in this area.

Keywords: Stretching; Hamstrings; Strains; Football; Attitudes; Beliefs; Football Coaches

\section{INTRODUCTION}

Soccer, or football, as it is officially called by the "Fédération Internationale de Football Association" (FIFA), is the most popular sport in the world. Due to the nature of the game, which involves kicking, sprinting and jumping, muscle strains are common and represent $33 \%$ of all injuries reported in the English professional football clubs [1]. Hamstring strains in particular are the most common type of muscular strain to affect the lower limb in the elite football player [2].

The literature suggests insufficient warm up, poor flexibility, muscle strength imbalance, muscle weakness, neural tension, muscle fatigue, dy-synergic contraction of muscle groups, previous injury and inadequate rehabilitation of previous injury [1,3] as predisposing factors for hamstring strains. Efforts should be made to prevent hamstring strain since it is a frustrating injury for coaches and football players: the symptoms are persistent, a significant amount of matches is missed per hamstring strain and re-injury rates are high [4]. Since muscle tightness is thought to predispose athletes to strains, stretching is widely regarded as an important part of warm-up and a necessity for fitness, flexibility and injury prevention [5]. However there is conflicting evidence in the literature concerning the relationship between stretching and 
injury prevention. Coaches usually recommend stretching before participation in sports activities in an effort to prevent injury [6] and football coaches are no exception. However, there is a relative lack of literature on the stretching practices that occur in professional sports [1]. Therefore the purpose of this study is to determine the attitudes, beliefs and practices of football coaches regarding stretching in the prevention of hamstring strains.

\section{METHODS}

The study sample consisted of a random sample of 35 football coaches in Mauritius who were registered with the MFA as at December-March 2010. The final sample consisted of 26 registered football coaches. The participants coached football teams either on a full-time or part-time basis.

\subsection{Survey Instrument}

The survey instrument used was a self-designed questionnaire based on a review of the literature and discussion with physiotherapists and football coaches otherwise not involved in this study. The questionnaire comprised three sections. Section A inquired about personal demographic information such as gender, age, number of years of experience as a football coach, and which team/ division was being coached. Section B consisted of an attitude scale to investigate the coaches' attitudes and beliefs towards stretching in football. A 5-point Likert scale was used, with "strongly disagree" and "strongly agree” as anchors. Ten positive and negative statements about stretching were interspersed in the scale. Finally Section $C$ inquired about the stretching protocols used on the field, the warm-up and weekly training patterns of football clubs, which techniques were used to stretch the hamstrings and the main reason that coaches advised players to stretch. The questionnaire consisted mostly of closed-ended questions with two open-ended questions in Section C.

The first draft of the questionnaire was piloted among 5 individuals and took approximately 15 minutes to be completed. As per the recommendations received from the pilot study, some technical terms had to be illustrated with diagrams or their equivalent French terms given in brackets.

\subsection{Conduct of Study}

Ethical clearance was granted by the Research Ethics Committee of the University of Mauritius for the study. After initial conversations on the phone, personal meetings were arranged between the researcher and the sports officers of the MFA. The sports officers agreed to allow the football coaches to participate in the study and they distributed the questionnaires to the football coaches. The questionnaires started being collected 2 weeks after initial distribution by the researcher. The final return rate was $77 \%$.

\subsection{Procedure}

The participants were given a booklet containing an information sheet, a consent form and a three-section questionnaire. The information sheet explained the aim of the study, provided definitions of key terms used throughout the questionnaire and the contact details of the researcher. Although the consent form included individual identifiers, it was explained to the sports officers that completing this part was optional.

\subsection{Data Analysis}

Initially response frequencies for each survey item were determined manually in tally form. Microsoft Office Excel 2007 was then used to analyse the data from the questionnaires. Where an item was left unanswered on the 5-point Likert scale, it was analysed as though a neutral response had been chosen [7]. In the other sections of the questionnaire, a valid percentage (i.e. the denominator is number of respondents to that specific question, not total number of participants in the study) was calculated wherever a question was left unanswered [8].

\section{RESULTS}

All the respondents were male, with an age range of 19 to 60 years old. The mean age was $46.0 \pm 10.6$ years. All had received formal training to become a football coach. The football coaches coached a variety of teams ranging from the National Team to the young players in so-called "Schools of Football". Table 1 shows the proportion of coaches and their corresponding years of experience.

\subsection{Attitudes and Beliefs towards Stretching}

The respondents held generally positive attitudes and beliefs towards stretching. A majority replied that they agreed or strongly agreed that: stretching before training or competition is beneficial (88\%) and that stretching before training or competition helps to prevent hamstring strains (93\%). A majority agreed or strongly agreed that tight hamstrings are a risk factor for hamstring strains (77\%) and that it is important for football players to have flexible muscles (100\%), as shown in Table 2. Respondents were diverse in their opinions about the statement that there was not enough scientific proof that stretching can reduce injuries: $46 \%$ stated they disagreed or strongly 
Table 1. Personal demographic information of football coaches.

\begin{tabular}{lcc}
\hline & $\begin{array}{c}\text { Number of } \\
\text { respondents }\end{array}$ & Percentage \\
\hline Years of experience as football coach & 6 & 24 \\
$<5$ years & 6 & 24 \\
5 - 10 years & 2 & 8 \\
10 - 15 years & 11 & 44 \\
$>15$ years & 9 & 36 \\
Full-time coaches & 16 & 64 \\
Part-time coaches & & \\
\hline
\end{tabular}

disagreed, $31 \%$ stated they agreed or strongly agreed with the statement and $23 \%$ were neutral.

\subsection{Perceived Benefits of Pre-Exercise Stretching}

A majority of coaches replied that they agreed or strongly agreed that: pre-exercise stretching (PES) helps to prevent hamstring strains (93\%), stretching acts as mental preparation for training/matches (77\%) and that PES increases a player's performance (88\%).

\subsection{Perceived Drawbacks of Stretching}

As shown in Table 2, responses were diverse concerning whether the time taken for stretching could be better used on more useful forms of preparation: 53\% stated they disagreed or strongly disagreed, 39\% stated they agreed or strongly agreed with this statement and $8 \%$ were neutral. Moreover $88 \%$ of respondents disagreed or strongly disagreed that stretching is harmful and can cause muscle injury.

\subsection{Training Patterns}

As shown in Table 3, coaches trained their teams on average for $15.1 \pm 7.5$ hours per week. Coaches devoted an average of $1.3 \pm 0.6$ hours per week to stretching/ flexibility training. As shown in Figure 1, football coaches devoted $31 \%$ of the total weekly training time to skills/ technical training, $30 \%$ to endurance training, $12 \%$ to strength/resistance training, and $10 \%$ to speed training. The time spent on stretching/flexibility training accounted for $9 \%$ of the total weekly training time.

All the coaches reported using warm-up before training sessions and before matches. The mean time for warm-up before training was $23 \pm 6$ minutes and the mean time for warm-up before matches was $26 \pm 8$ minutes.

Figure 2 shows the time at which stretching is recommended by coaches. The majority of coaches recommended stretching after warming up (81\%) and after the training session (93\%). 22\% reported recommending stretching before warm-up, 33\% during warm-up and 26\% recommended stretching at mid-session. $76 \%$ of coaches also recommended stretching outside training sessions e.g. at home.

\subsection{Stretching of the Hamstrings}

$96 \%$ of coaches reported using the static stretching technique to stretch the hamstrings while $25 \%$ used the ballistic technique and $42 \%$ the PNF technique. $46 \%$ reported using more than one technique to stretch the hamstrings, and when this was the case, static stretching (64\%) was reported as being the most used technique.

Coaches using static stretching for the hamstrings held the stretch on average for $12 \pm 3$ seconds(s). As shown in Figure 3, 10\% held the stretch between 5 - 9 s, 61\% between 10 - 14 s, 24\% between 15 - 19 s and $5 \%$ for $20 \mathrm{~s}$ or more. The number of repetitions ranged from 1 to 7 ,

Table 2. Attitudes towards stretching.

\begin{tabular}{|c|c|c|c|c|c|}
\hline & $\begin{array}{c}\text { Strongly } \\
\text { Disagree (\%) }\end{array}$ & Disagree (\%) & Neutral (\%) & Agree (\%) & $\begin{array}{c}\text { Strongly Agree } \\
(\%)\end{array}$ \\
\hline $\mathrm{PES}^{*}$ is beneficial & 0 & 4 & 8 & 50 & 38 \\
\hline PES $^{*}$ prevents hamstring strains & 0 & 0 & 8 & 62 & 31 \\
\hline Time taken on stretching could be better spent & 15 & 38 & 8 & 31 & 8 \\
\hline Warm-up is more important than stretching & 4 & 15 & 19 & 54 & 8 \\
\hline Hamstring tightness is a risk factor for strains & 0 & 12 & 12 & 69 & 8 \\
\hline Stretching can cause muscle injury & 4 & 12 & 8 & 73 & 4 \\
\hline Flexibility is important for football players & 42 & 46 & 8 & 4 & 0 \\
\hline Lack of scientific proof that stretching reduces injuries & 0 & 4 & 8 & 73 & 15 \\
\hline
\end{tabular}

${ }^{*}$ PES $=$ pre-exercise stretching. 


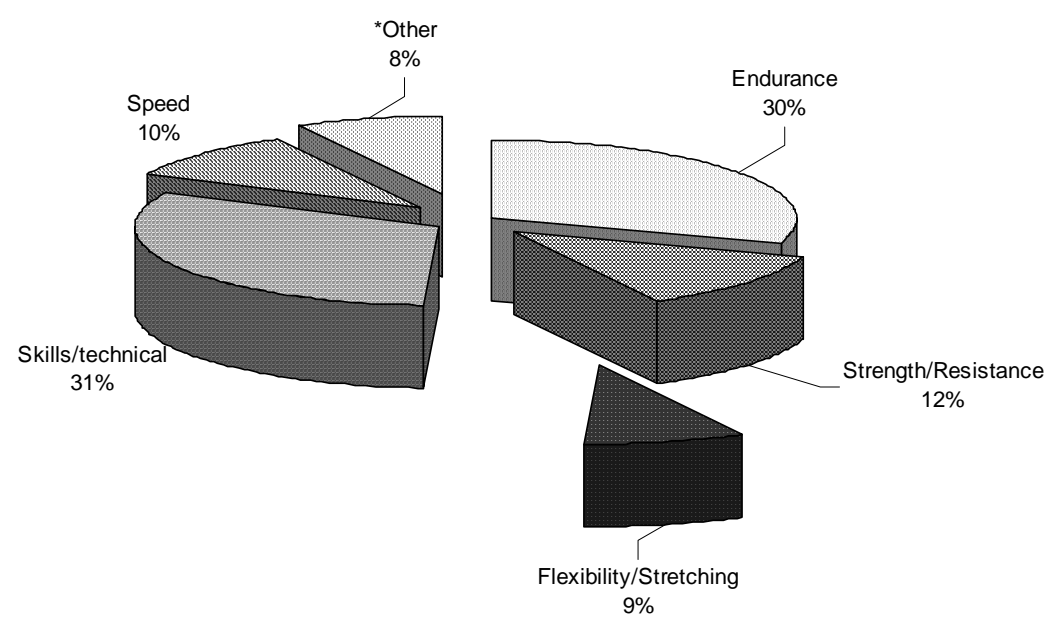

Figure 1. The proportion of each training modality in football clubs. ('Other includes verbal briefing, moral support, explaining tactics and strategies of the game and mental training).

Table 3. The distribution of weekly training in football clubs.

\begin{tabular}{ccc}
\hline Training modality & $\begin{array}{c}\text { Total } \\
\text { (hours/week) }\end{array}$ & $\begin{array}{c}\text { Mean } \pm \text { SD } \\
\text { (hours/week) }\end{array}$ \\
\hline Endurance training & 109.50 & $4.6 \pm 3.8$ \\
Strength/resistance training & 43.50 & $1.8 \pm 0.3$ \\
Flexibility/stretching & 31.50 & $1.3 \pm 0.6$ \\
Skills/technical training & 114.00 & $4.8 \pm 3.0$ \\
Speed training & 36.25 & $1.5 \pm 1.1$ \\
Other & 28.75 & $1.7 \pm 1.2$ \\
Total & 363.50 & $15.1 \pm 7.5$ \\
\hline
\end{tabular}

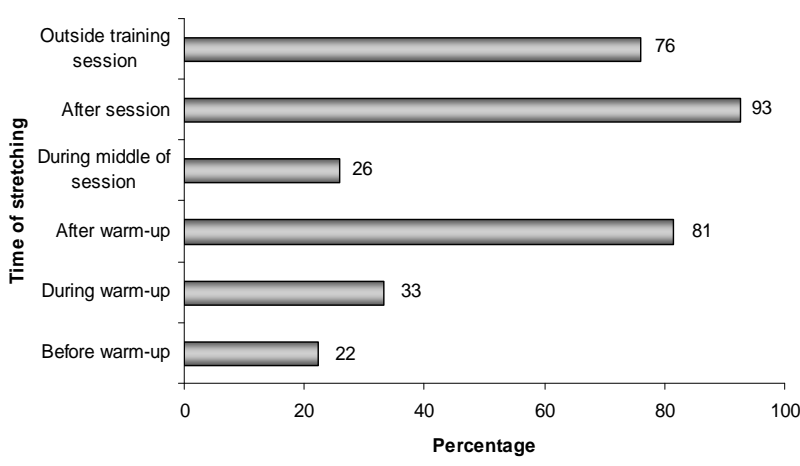

Figure 2. The time at which stretching is recommended by coaches.

with an average of $4 \pm 1$. Hence football players stretch the hamstring muscles of each leg for $48 \mathrm{~s}$ on average at each training session.

\subsection{Reason for Advising Stretching}

The open-ended question asking coaches why they

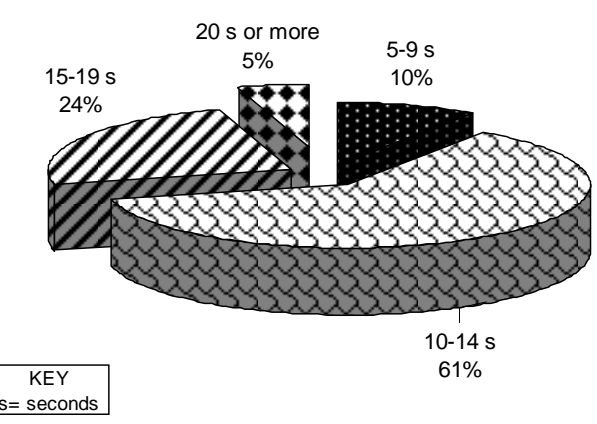

Figure 3. The duration of a static stretch for the hamstrings.

advised players to stretch revealed that answers had a tendency to fall into 4 main categories. 91\% wrote that they advised stretching to avoid muscle injury or to avoid injuries, $17 \%$ to prepare the muscle for exertion, $13 \%$ to prevent muscle cramps and $13 \%$ to increase flexibility, as shown in Figure 4.

\subsection{Nordic Hamstring Exercises}

$73 \%$ of the coaches reported being familiar with the Nordic hamstring exercise. Of those that were familiar with this exercise, when asked whether they believed the Nordic hamstring exercise was more useful than stretching in preventing hamstring strains, $63 \%$ of these coaches agreed and 37\% were "undecided".

\subsection{Influence on Stretching \\ Recommendations}

When asked which factors would most likely influence their stretching recommendations, football coaches more often answered that they would be influenced by personal experience (69\%), scientific research $(81 \%)$ and 


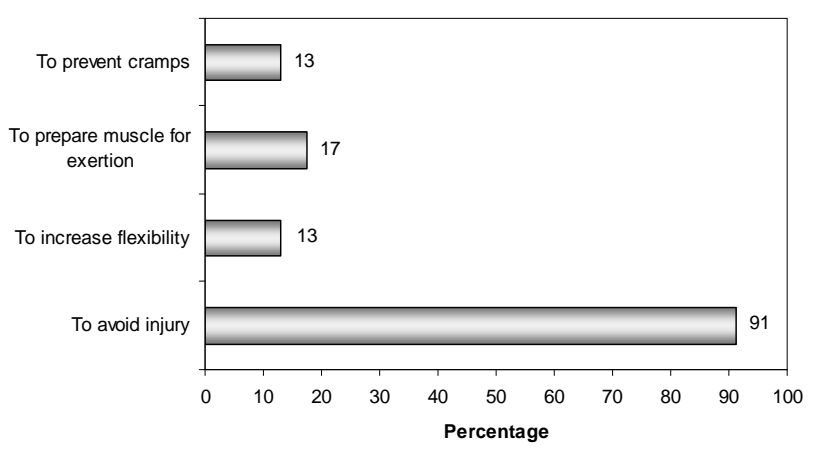

Figure 4. The reasons stated by coaches for advising stretching.

FIFA guidelines (81\%) as shown in Figure 5.

\subsection{Other Techniques to Prevent Hamstring Strains}

The open-ended question about any other techniques used by football coaches to prevent hamstring strains (apart from stretching, warm-up and eccentric exercise) yielded answers as shown in Figure 6. Popular answers were massage (20\%) and swimming/aquagym (20\%). 7\% answered "leg curls" which has been categorised as concentric hamstring exercise as this exercise focuses on the concentric phase, although leg curls do have an eccentric hamstring component. Another 7\% answered "hack squats” which has been classified as quadriceps strengthening and 3\% answered cycling. 7\% gave answers such as drinking a lot of water and horse riding, which were grouped under the category "others".

\section{DISCUSSION}

\subsection{Attitudes and Beliefs towards Stretching}

Results from the study showed that football coaches who are members of the Mauritius Football Association (MFA) have generally positive attitudes and beliefs regarding stretching. These attitudes and beliefs are similarly reflected in a study on high school coaches in the USA [8]. From the findings, the vast majority believed that stretching before training or competition helps to prevent hamstring strains (93\%). However, the literature contains limited evidence to support the hypothesis that PES can reduce injuries sustained during training or competition. Many systematic reviews of the literature have concluded that PES does not significantly reduce exercise-related injuries [6,9-11]. However, it must be noted that these reviews were not based exclusively on studies that investigated the effect of PES on hamstring strains in football players. There is a lack of studies on the effect of stretching on hamstring strains in football players [12]. The effect of contract-relax PNF stretching

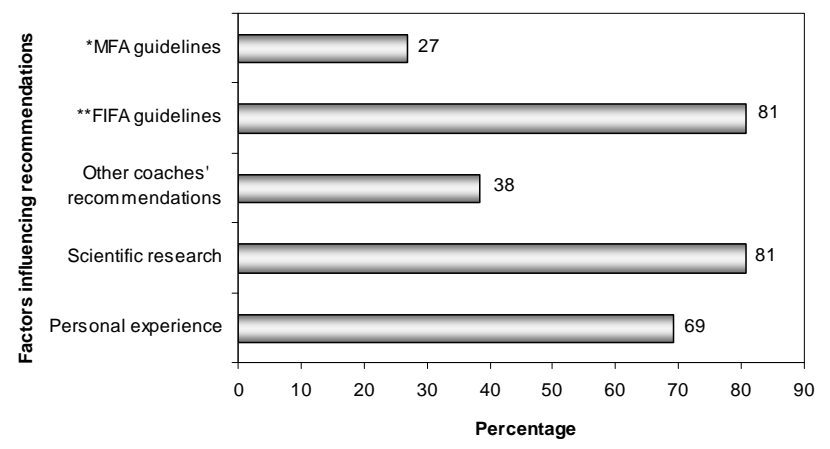

Figure 5. The factors most likely to influence coaches' stretching recommendations ( ${ }^{*} \mathrm{MFA}=$ Mauritius Football Association; ${ }^{* *}$ FIFA = Fédération Internationale de Football Association).

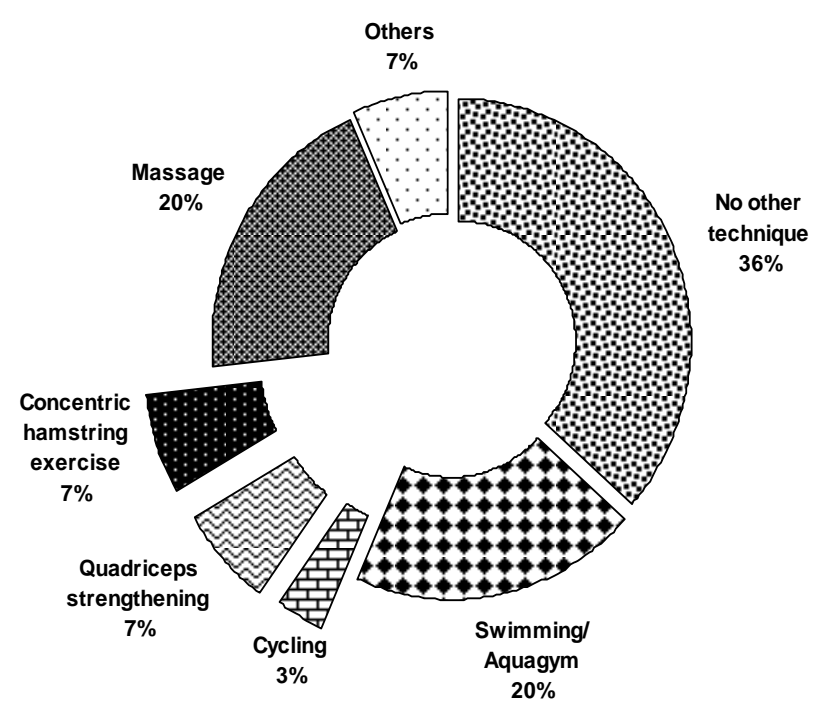

Figure 6. Other techniques to prevent hamstring muscle strain.

before training in a football population has been studied [13]. Although the intervention significantly decreased football injuries, the efficacy of the stretching procedure itself could not be evaluated due to the multiple interventions used. In another study, a pre-exercise static stretching programme significantly reduced the number of strains of the lower limb in American football players [14]. Other studies carried out on army recruits and on recreational runners have not found significant decreases in injuries after the implementation of a pre-exercise static stretching (PES) protocol $[15,16]$. Therefore there is insufficient scientific evidence to support the MFA coaches' beliefs that PES can reduce hamstring strains. In fact, $31 \%$ of coaches agreed that there is a lack of scientific proof on the subject matter while $46 \%$ disagreed or strongly disagreed to this statement. This suggests that football coaches who felt that there was a lack of evidence on the effectiveness of stretching in decreasing muscle strains nevertheless used stretching, probably out of habit or based on personal experience. 
From the findings, $88 \%$ of coaches believed that PES increases a player's performance. Evidence in the literature tends to point towards a decrease in strength, and thus in performance, following an acute bout of stretching $[17,18]$. Some authors found that a bout of static stretching decreased maximal voluntary force for up to two hours $[19,20]$. On the other hand, dynamic stretching protocols may be beneficial for explosive type of activities [21] and may be useful for increasing flexibility without decreasing athletic performance [22].

From the study, it was found that $77 \%$ of the football coaches believed that tight hamstrings are a risk factor for hamstring strains and all the respondents believed that it is important for football players to have flexible muscles. It has been suggested that football players with hamstring tightness should stretch intensively so as to decrease their risk of muscle strain [23]. Increased flexibility (i.e. more compliant muscle) may help to reduce the risk of injury [22] and may benefit performance in football which involves a high intensity of stretch-shortening cycles [24].

\subsection{Stretching Practices}

MFA coaches stretched their players on average for $1.3 \pm 0.6$ hours per week. This time period included stretching of the hamstrings as well as all other muscles that football coaches stretch. $76 \%$ of MFA football coaches also recommended stretching outside training sessions, and if football players follow their coaches' recommendations, the amount of flexibility training would therefore be more than the reported 1.3 hours per week. We did not investigate the duration of stretching recommended by coaches outside training sessions. Although appropriate stretching protocols are thought to increase flexibility, the optimal level of flexibility that prevents injury is not clear and is thought to vary between muscle groups and probably sports [1]. The recommended flexibility training protocol for the hamstrings is to stretch them for 5 - 10 minutes at a time, at least three times per week during the preseason period and twice a week during the competitive season [25]. The majority of coaches advised stretching before the training session. It has been suggested that rather than stretching prior to exercise, stretching should be done after the training session or outside the training environment, such as at home [26]. Stretching regularly outside the training environment may be beneficial and help reduce injuries, in contrast with PES [11,26].

In this study, static stretching was reported as being the most used technique to stretch the hamstrings. The popularity of static stretching may be explained by its simplicity of application [22]. PNF stretching alone and ballistic stretching alone were less popular among foot- ball coaches. Nearly half of the football coaches used more than one technique to stretch the hamstrings, usually a combination of static stretching and PNF stretching was used. These practices parallel those of English professional football clubs, where static stretching was reported as being the most popular technique to be used, and that it was also commonly used in combination with PNF stretching [1]. 25\% of the coaches used ballistic stretching for the hamstrings although it has been suggested in the literature that ballistic stretching has more potential than other stretching techniques to cause injury to the muscle [22].

The survey revealed that coaches using static stretching for the hamstrings held the stretch on average for 12 \pm 3 seconds and performed an average of $4 \pm 1$ repetitions. The majority advised a static stretch holding time of 10 - 14 seconds. Changes in viscoelasticity of musculotendinous units depend more on the duration of stretch rather than the number of stretches or the length of the stretching training period [22]. Previous studies tend to suggest that a static stretch holding time of 15 to 30 seconds is the most effective for gaining muscle flexibility [1]. Other authors suggested that a long stretch duration of up to 90 seconds might be most effective in increasing musculotendinous unit compliance [22]. Therefore coaches are using a stretch holding time inferior to that which is recommended in order to increase flexibility and thus to gain any risk-reducing benefit from the stretching process.

A large proportion of the respondents indicated that their recommendations regarding stretching would most likely be influenced by scientific research and by FIFA guidelines. This suggests that MFA coaches are willing to incorporate evidence into practice and encourages further research in this area. If the evidence is made available through FIFA publications, MFA coaches may more readily put them into practice.

\subsection{Other Modalities to Prevent Hamstring Strains}

Eccentric training of the hamstrings has been shown to decrease hamstring injuries in elite football players [12]. Therefore it can be suggested that hamstring strains can be reduced with an appropriate eccentric exercise protocol [27]. When asked whether they knew any other modality that could prevent hamstring strains (apart from stretching, warm-up and eccentric exercise), $36 \%$ of MFA coaches did not give any answer. The most popular answers were massage (20\%) and swimming or aquagym (20\%). Massage is sometimes included in warm-up protocols but its effect has not been widely studied [28]. Swimming, aquagym and cycling can be classified as aerobic or cardiovascular training. It has been suggested 
that athletes should better invest their time in cardiovascular training, rather than in stretching as there is some evidence that the risk of injury to muscles is more closely related to poor aerobic fitness than to lack of flexibility [29].

$7 \%$ of the coaches reported using leg curls as a modality for preventing hamstring strains. Leg curls are also known as hamstring curls and are the most commonly used concentric strength training exercise for the hamstrings [30]. Nordic hamstring exercise was found to be more effective than hamstring curls in developing maximal eccentric hamstring strength, the hamstring: quadriceps strength ratio (H:Q ratio) and isometric hamstring strength [30]. The Nordic hamstring exercise may thus be more effective than hamstring curls in reducing the risk of sustaining a hamstring strain [30]. A further 7\% of coaches proposed hack squat exercises to help prevent hamstring strains. Hack squats strengthen primarily the quadriceps muscles. A strengthening programme of the quadriceps must be accompanied by strengthening of the hamstrings, since lowering the $\mathrm{H}: \mathrm{Q}$ ratio may increase the risk of a hamstring strain $[1,3]$.

\section{CONCLUSIONS}

Despite the lack of conclusive evidence, the vast majority of football coaches registered with the MFA believed that PES is beneficial and that it helps to prevent hamstring strains. They also believed that PES increases a player's performance, although recent evidence tends to show a reduction in performance after a bout of stretching. The coaches agreed that it is important for football players to have flexible muscles and they also recommended stretching outside the training sessions.

There is insufficient evidence to make definitive recommendations regarding PES for the prevention of hamstring strains in football players. However it is suggested that stretching regularly outside the training environment may have different effects from PES and that it may be beneficial. Increasing flexibility and thus muscle compliance through a regular stretching programme that is not performed prior to training may help reduce the risk of muscle injury and may benefit performance in football players. Coaches reported that their stretching practices would be most likely influenced by FIFA guidelines and by scientific research. Thus there is potential for further research in this area.

\section{RECOMMENDATIONS}

Randomised controlled trials are needed to determine the effects of PES and regular stretching outside training on injury prevention, and especially on hamstring strains in football players. Moreover, future research on stretching practices could include the whole population of foot- ball coaches registered with the MFA. Independent data collection, rather than self-reporting of the stretching practices of coaches could give more accurate results. Coaches should be encouraged to search for relevant literature and to incorporate evidence into their practice. Coaches should be aware of the modalities that are available to prevent hamstring strains in football players such as appropriate warm-up, regular long-term stretching after training or outside the exercise environment, eccentric training of the hamstring muscles and cardiovascular training. First-time hamstring strains should be properly rehabilitated so as to avoid re-injury.

Before training sessions or matches, coaches may use "The 11+" which is a comprehensive warm-up programme designed by the FIFA for football players. Coaches wishing to stretch prior to training may use dynamic stretching after the warm-up routine rather than static or ballistic stretching. Coaches may continue to advise players to use static or proprioceptive neuromuscular facilitation stretching after training sessions and regularly at home.

\section{ACKNOWLEDGEMENTS}

We wish to thank all the coaches who participated in the study and the University of Mauritius.

\section{REFERENCES}

[1] Dadebo, B., White, J. and George, K.P. (2004) A survey of flexibility training protocols and hamstring strains in professional football clubs in England. British Journal of Sports Medicines, 38, 388-394. doi:10.1136/bjsm.2002.000044

[2] Askling, C. (2008) Hamstring muscle strain. Ph.D. Thesis, Karolinska Institute, Solna.

[3] Woods, C. Hawkins, R.D. Maltby, S. Hulse, M. Thomas, A. and Hodson, A. (2004) The Football Association Medical Research Programme: An audit of injuries in professsional football-analysis of hamstring injuries. British Journal of Sports Medicines, 38, 36-41. doi:10.1136/bjsm.2002.002352

[4] Petersen, J. and Holmich, P. (2005) Evidence based prevention of hamstring injuries in sport. British Journal of Sports Medicines, 39, 319-323. doi:10.1136/bjsm.2005.018549

[5] Smith, C.A. (1994) The warm-up procedure: To stretch or not to stretch. A brief review. Journal Orthopaedic \& Sports Physical Therapy, 19, 12-17.

[6] Thacker, S.B., Gilchrist, J. Stroup, D.F. and Kimsey, C.D, (2004) The impact of stretching on sports injury risk: A systematic review of the literature. Medical Science Sports Exercise, 36, 371-378. doi:10.1249/01.MSS.0000117134.83018.F7

[7] Polit, D.F. and Beck, C.T. (2003) Nursing research: Principles and Methods. 7th Edition, Lippincott Williams and Wilkins, Philadelphia. 
[8] Shehab, R., Mirabelli, M. Gorenflo, D. and Fetters, M.D. (2006) Pre-exercise stretching and sports related injuries: Knowledge, attitudes and practices. Clinical Journal Sport Medicines, 16, 228-231. doi:10.1097/00042752-200605000-00007

[9] Andersen, J.C., (2005). Stretching before and after exercise: Effect on muscle soreness and injury risk. Journal of Athletes Training, 40, 218-220.

[10] Herbert, R.D. and Gabriel, M. (2002) Effects of stretching before and after exercising on muscle soreness and risk of injury: Systematic review. British Medical Journal, 325, 1-5.

[11] Shrier, I. (2007) Does stretching help prevent injuries? In Macauley, D. and Best T.M. Eds., Evidence-based sports medicine, 2nd Edition, Blackwell Publishing, London, 36-58. doi:10.1002/9780470988732.ch3

[12] Arnason A., Andersen, T.E, Holme, I, Engebretsen, L. and Bahr, R. (2007) Prevention of hamstring strains in elite soccer: An intervention study. Scandinavian Journal of Medicines \& Science Sports, 18, 40-48. doi:10.1111/j.1600-0838.2006.00634.X

[13] Ekstrand, J., Gillquist J. and Liljedahl S.O. (1983) Prevention of soccer injuries: supervision by doctor and physiotherapist. American Journal Sports Medicines, 11, 116120. doi:10.1177/036354658301100302

[14] Cross, K.M. and Worrell, T.W. (1999) Effects of a static stretching program on the incidence of lower extremity musculotendinous strains. Journal Athletic Training, 34, 11-14.

[15] Pope, R.P., Herbert, R.D., Kirwan, J.D. and Graham, B.J. (2000) A randomised trial of preexercise stretching for prevention of lower-limb injury. Medical Science Sports Exercise, 32, 271-277. doi:10.1097/00005768-200002000-00004

[16] Van Mechelen., W., Hlobil, H. Kemper, H.C.G., Voorn, W.J. and De Jongh, R. (1993) Prevention of running injuries by warm-up, cool-down, and stretching exercises. American Journal Sports Medicines, 21, 711-719. doi:10.1177/036354659302100513

[17] Gremion, G. (2005) The effect of stretching on sports performance and the risk of sports injury: A review of the literature. Sportmedizin und Sporttraumatologie, 53, 6-10.

[18] Rubini, E.C., Costa, A.L.L and Gomes, P.S.C. (2007) The effects of stretching on strength performance. Sports Medicines, 37, 213-224. doi:10.2165/00007256-200737030-00003

[19] Fowles, J.R. Sale, D.G. and Macdougall, J.D. (2000) Re- duced strength after passive stretch of the human plantarflexors. Journal of Applied Physiology, 89, 1179-1188.

[20] Power, K., Behm, D. Cahill, F. Carroll, M. and Young, W. (2004) An acute bout of static stretching: effects on force and jumping performance. Medical Science Sports Exercise, 36, 1389-1396.

doi:10.1249/01.MSS.0000135775.51937.53

[21] Little, T. and Williams A.G. (2006) Effects of differential stretching protocols during warm-ups on high-speed motor capacities in professional soccer players. Journal Strength Condition Research, 20, 203-207.

[22] Weerapong, P., Hume, P.A. and Kolt, G.S. (2004) Stretching: Mechanisms and benefits for sport performance and injury prevention. Physical Therapy Reviews, 9, 189-206. doi:10.1179/108331904225007078

[23] Witvrouw, E., Danneels, L. Asselman, P. D’Have, T. and Cambier, D. (2003) Muscle flexibility as a risk factor for developing muscle injuries in male professional soccer players. A prospective study. American Journal Sports Medicine, 31, 41-46.

[24] Witvrouw, E., Mahieu, N. Danneels, L. and Mcnair, P. (2004) Stretching and injury prevention: An obscure relationship. Sports Medicine, 34, 443-449. doi:10.2165/00007256-200434070-00003

[25] Dvorak, J., and Junge, A. (2005). F-MARC football medicine manual. FIFA, Zurich.

[26] Shrier, I. (2004) Does stretching improves performance? A systematic and critical review of the literature. Clinical Journal Sports Medicines, 14, 267-273. doi:10.1097/00042752-200409000-00004

[27] Brughelli, M. and Cronin, J. (2008) Preventing hamstring injuries in sport. Strength and Conditioning Journal, 30, 55-64. doi:10.1519/SSC.0b013e3181638263

[28] Goats, G.C. (1994) Massage-The scientific basis of an ancient art: Part 2. Physiological and therapeutic effects. British Journal Sports Medicines, 28, 153-156. doi:10.1136/bjsm.28.3.153

[29] Ingraham, S.J. (2003) The role of flexibility in injury prevention and athletic performance. Have we stretched the truth? Minnesota Medicine, 86, 58-61. doi:10.1046/j.1600-0838.2003.367.x

[30] Mjølsnes, R., Arnason, A, Østhagen, T, Raastad, T. and Bahr, R. (2004) A 10-week randomized trial comparing eccentric vs. concentric hamstring strength training in welltrained soccer players. Scandinavian Journal of Medicine \& Science, 14, 311-317. 